\title{
Deducing the Constraints in the Light-Cone $S U$ (3) Yang-Mills Mechanics Via Gröbner Bases
}

\author{
Vladimir Gerdt ${ }^{1}$, Arsen Khvedelidze ${ }^{1,2}$, and Yuri Palii ${ }^{1,3}$ \\ ${ }^{1}$ Laboratory of Information Technologies, Joint Institute for Nuclear Research, \\ Dubna, Moscow Region, 141980, Russia \\ gerdt@jinr.ru \\ 2 Department of Theoretical Physics, A.Razmadze Mathematical Institute, \\ Tbilisi, GE-0193, Georgia \\ akhved@jinr.ru \\ ${ }^{3}$ Institute of Applied Physics, Moldova Academy of Sciences, \\ Chisinau, MD-2028, Republic of Moldova \\ palii@jinr.ru
}

\begin{abstract}
The algorithmic methods of commutative algebra based on the Gröbner bases technique are briefly sketched out in the context of an application to the constrained finite dimensional polynomial Hamiltonian systems. The effectiveness of the proposed algorithms and their implementation in Mathematica is demonstrated for the light-cone version of the $S U(3)$ Yang-Mills mechanics. The special homogeneous Gröbner basis is constructed that allow us to find and classify the complete set of constraints the model possesses.
\end{abstract}

\section{Introduction}

The basic procedure, completion to involution 123456, of systems of differential equations represents a highly nontrivial issue in view of its practical application. Particularly, a manipulation with functions modulo a set of algebraic relations requires an efficient algorithmization and implementation in a proper computer algebra software. For the practical purposes of wide class of theories and models of the contemporary theoretical and mathematical physics and especially of the degenerate Hamiltonian systems [7]-[9] the problem of completion to involution being very topical became nowadays feasible due to the progress in computer technologies. Our attempts to implement such an algorithmic description for the degenerate polynomial Hamiltonian mechanical models have been summarized in the recent papers [10-[14, where the method based on the most universal algorithmic tool of commutative algebra, the well-known Gröbner bases theory [15- 17, has been elaborated. Since this technique provides an effective algorithmic instrument to verify whether a polynomial vanishes on the manifold defined by a set of other polynomials, the Gröbner bases plays the principal role in algorithmic implementation of the basic operations of the Dirac constraint formalism: computation and separation of constrains.

V.G. Ganzha, E.W. Mayr, and E.V. Vorozhtsov (Eds.): CASC 2007, LNCS 4770, pp. $145-1592007$. (C) Springer-Verlag Berlin Heidelberg 2007 
Here we briefly sketch out this very central element of the Dirac-BergmannGröbner algorithmic procedure suggested in [10] to deal with the practically important case of finite-dimensional degenerate polynomial Lagrangian system. Afterwards we apply this algorithm to examine the mechanical system with a rich set of constraints, the so-called light-cone $S U(3)$ Yang-Mills mechanics, where computation of constraints has not been done before.

The outline of the article is as follows. In section 2 the basic elements of the Dirac-Bergmann-Gröbner algorithm to compute and classify the constraints are given. Then the formulation of the mechanical model, the light-cone $S U(3)$ Yang-Mills mechanics is presented. In section 3 the results of computation of the complete set of constraints are given. The section 4 is devoted to the discussion of the specially constructed homogeneous Gröbner basis that provides our calculations and categorization of the constraints.

\section{Elements of the Dirac-Bergmann-Gröbner Algorithm}

The Dirac method to determine and classify constraints for degenerate Hamiltonian systems is easy formulate but difficult to implement at practical level of computation when the both, number of degrees of freedom as well as the number of free parameters of the model are sufficiently large. Here we describe a possible way to make this procedure computationally effective. We start with the discussion of the Dirac constraint formalism for a finite dimensional degenerate Lagrangian system aiming its algorithmic reformulation.

Consider an $n$-dimensional mechanical system whose configuration space is $\mathbf{R}^{n}$ and the Lagrangian $L(q, \dot{q})$ is defined on a tangent space as a function of the coordinates $q:=q_{1}, q_{2}, \ldots, q_{n}$ and velocities $\dot{q}:=\dot{q}_{1}, \dot{q}_{2}, \ldots, \dot{q}_{n}$.

The Lagrangian system is regular if the rank $r:=\operatorname{rank}\left\|H_{i j}\right\|$ of the corresponding Hessian function $H_{i j}:=\partial^{2} L / \partial \dot{q}_{i} \partial \dot{q}_{j}$ is maximal $(r=n)$. In this case the Euler-Lagrange equations

$$
\frac{\mathrm{d}}{\mathrm{d} t}\left(\frac{\partial L}{\partial \dot{q}_{i}}\right)-\frac{\partial L}{\partial q_{i}}=0, \quad 1 \leq i \leq n
$$

rewritten explicitly as

$$
H_{i j} \ddot{q}_{j}+\frac{\partial^{2} L}{\partial q_{j} \partial \dot{q}_{i}} \dot{q}_{j}-\frac{\partial L}{\partial q_{i}}=0
$$

can be resolved with respect to the accelerations $(\ddot{q})$ and there are no hidden constraints. Otherwise, if $r<n$, the Euler-Lagrange equations (and, thus, the Lagrangian system itself) are degenerate or singular. In this case not all differential equations (1) are of second order, namely there are $n-r$ independent equations, Lagrangian constraints, containing only coordinates and velocities. Passing to the Hamiltonian description via a Legendre transformation

$$
p_{i}:=\frac{\partial L}{\partial \dot{q}_{i}}
$$


the degeneracy of the Hessian results in the existence of $n-r$ relations between coordinates and momenta, the primary constraints

$$
\varphi_{a}^{(1)}(p, q)=0, \quad 1 \leq a \leq n-r
$$

Equations (3) define the so-called primary constraints subset (manifold, if certain regularity conditions assumed) $\Sigma_{1} 1$ This definition is implicit and therefore it is necessary to provide an effective algorithm to compute all primary constraints describing the subset $\Sigma_{1}$.

From (3) the dynamics is constrained by the set $\Sigma_{1}$ and by the Dirac prescription is governed by the total Hamiltonian

$$
H_{T}:=H_{C}+U_{a} \varphi_{a}^{(1)}
$$

which differs from the canonical Hamiltonian $H_{C}(p, q)=p_{i} \dot{q}_{i}-L$ by a linear combination of the primary constraints with the Lagrange multipliers $U_{a}$.

The next step is to analyze the dynamical requirement that classical trajectories remain in $\Sigma_{1}$ during the evolution

$$
\dot{\varphi}_{a}^{(1)}=\left\{H_{T}, \varphi_{a}^{(1)}\right\} \stackrel{\Sigma_{1}}{=} 0 .
$$

In (5) the evolutional changes are generated by the canonical Poisson brackets with the total Hamiltonian (4) and the abbreviation $\stackrel{\Sigma_{1}}{=}$ stands for a week equality, i.e., the right-hand side of (5) vanishes modulo the primary constraints (3).

The consistency condition (5), unless it is satisfied identically, may lead either to a contradiction or to a determination of the Lagrange multipliers $U_{a}$ or to new constraints. The former case indicates that the given Hamiltonian system is inconsistent.

In the latter case when (5) is not satisfied identically and is independent of the multipliers $U_{a}$ the left-hand side of (5) defines the new constraints. Otherwise, if the left-hand side depends on some Lagrange multipliers $U_{a}$ the consistency condition determines these multipliers, and, therefore, the constraints set is not enlarged by new constraints. The subsequent iteration of this consistency check ends up with the complete set of constraints and/or determination of some/or all Lagrange multipliers.

The number of Lagrange multipliers $U_{a}$ which can be found is determined by the rank of the so-called Poisson bracket matrix

$$
\mathbf{M}_{\alpha \beta}: \stackrel{\Sigma}{=}\left\{\phi_{\alpha}, \phi_{\beta}\right\}
$$

where $\Sigma$ denotes the subset of a phase space defined by the complete set of constraints $\Phi:=\left(\phi_{1}, \phi_{2}, \ldots, \phi_{k}\right)$

${ }^{1}$ Everywhere in this paper we suppose that all constraints satisfy the so-called regularity conditions (see explanations in $\S 1.1 .2$ of 9 ). 


$$
\Sigma: \quad \phi_{\alpha}(p, q)=0, \quad 1 \leq \alpha \leq k .
$$

including all primary $\varphi^{(1)}$, secondary $\varphi^{(2)}$, ternary $\varphi^{(3)}$, etc., constraints,

If $\operatorname{rank}(\mathbf{M})=m$, then $s:=k-m$ linear combinations of constraints $\phi_{\alpha}$

$$
\psi_{\alpha}(p, q)=\sum_{\beta} \mathrm{c}_{\alpha \beta}(p, q) \phi_{\beta},
$$

define the first-class constraints, whose Poisson brackets are weakly zero

$$
\left\{\psi_{\alpha}(p, q), \psi_{\beta}(p, q)\right\} \stackrel{\Sigma}{=} 0 \quad 1 \leq \alpha, \beta \leq s .
$$

The remaining functionally independent constraints form the subset of secondclass constraints.

It is worth to note here that the described method to find constraints within the Dirac formalism represent the reformulation of completion of the initial Hamiltonian equations to involution in another words and constraints corresponds to a set of the integrability conditions [181920].

Now the algorithmic reformulation of the above stated scheme will be described using the ideas and the terminology of the Gröbner bases theory. In doing so, we restrict our consideration to an arbitrary dynamical system with finitely many degrees of freedom whose Lagrangian is a polynomial in coordinates and velocities with rational (possibly parametric) coefficients $L(q, \dot{q}) \in \mathbf{Q}[q, \dot{q}]$. Thereafter we use the standard notions and definitions of commutative algebra (see, e.g., 15[16[17]).

\section{Algorithm to determine the primary constraints}

The primary constraints (3) are consequences of the polynomial relations (2). These relations generate the polynomial ideal in $\mathbf{Q}[p, q, \dot{q}]$

$$
I_{p, q, \dot{q}} \equiv \operatorname{Id}\left(\cup_{i=1}^{n}\left\{p_{i}-\partial L / \partial \dot{q}_{i}\right\}\right) \subset \mathbf{Q}[p, q, \dot{q}] .
$$

Thereby, primary constraints (3) belong to the radical $\sqrt{I_{p, q}}$ of the elimination ideal

$$
I_{p, q}=I_{p, q, \dot{q}} \cap \mathbf{Q}[p, q] .
$$

Correspondingly, for an appropriate term ordering which eliminates $\dot{q}$, a Gröbner basis of $I_{p, q}$ (denotation: $G B\left(I_{p, q}\right)$ ) is given by [151617]

$$
G B\left(I_{p, q}\right)=G B\left(I_{p, q, \dot{q}}\right) \cap \mathbf{Q}[p, q] .
$$

This means that construction of the Gröbner basis for the ideal (10) with omitting elements in the basis depending on velocities and then constructing of $G B\left(I_{p, q}\right)$ allows to compute the set of primary constraints. If $G B\left(I_{p, q}\right)=\emptyset$, then the dynamical system is regular. Otherwise, the algebraically independent set $\Phi_{1}$ of (effective) primary constraints can be found as the subset $\Phi_{1} \subset G B\left(\sqrt{I_{p, q}}\right)$ such that

$$
\forall \phi(p, q) \in \Phi_{1}: \phi(p, q) \notin \operatorname{Id}\left(\Phi_{1} \backslash\{\phi(p, q)\}\right)
$$


Verification of (11) is algorithmically done by computing the following normal form: $N F\left(\phi, G B\left(\operatorname{Id}\left(\Phi_{1} \backslash\{\phi\}\right)\right)\right.$. In addition, the canonical Hamiltonian $H_{c}(p, q)$ is computed as $N F\left(p_{i} \dot{q}_{i}-L, G B\left(I_{p, q, \dot{q}}\right)\right)$. This form of $H_{c}(p, q)$ is used in the next steps of the Dirac-Bergman-Gröbner algorithm.

\section{Algorithm to determine the higher constraints and to classify them}

The dynamical consequences (5) of a primary constraint can also be algorithmically analyzed by computing the normal form of the Poisson brackets of the primary constraint and the total Hamiltonian modulo $\sqrt{I_{p, q}}$ ). Here the Lagrange multipliers $U_{a}$ in (4) are treated as time-dependent functions. If the non-vanishing normal form does not contain $U_{a}$, then it is nothing else than the secondary constraint. In this case the set of primary constraints is enlarged by the secondary constraint obtained and the process is iterated. At the end either the complete set $\Phi$ of constraints (7) is constructed or some inconsistency is detected. The detection holds when the intermediate Gröbner basis, whose computation is a part of the iterative procedure, becomes $\{1\}$.

In order to separate the set $\Phi=\left\{\phi_{1}, \ldots, \phi_{k}\right\}$ into subsets of the first and second classes constraints the entries of Poisson brackets matrix $\mathbf{M}$ are evaluated as normal forms of the Poisson brackets of the constraints modulo a Gröbner basis of the ideal generated by set $\Phi$. Afterwards if the basis $E=\left\{\mathbf{e}^{(1)}, \ldots, \mathbf{e}^{(k-m)}\right\}$ of the null space (kernel) of this matrix $\mathbf{M}$ is known the each basis vector $\mathbf{e}^{(s)} \in E, s=1, \ldots, k-m$ generates the first-class constraint of form $\mathbf{e}_{\alpha}^{(s)} \phi_{\alpha}$. The second class constraints are build using the basis of the $m$-dimensional orthogonal complement $E_{\perp}$, of subspace $E$. With the aid of these vectors $\mathbf{e}_{\perp}^{(l)} \in E_{\perp}, l=1, \ldots, m$ the second-class constraint are constructed as $\mathbf{e}_{\perp \alpha}^{(l)} \phi_{\alpha}$.

Concluding we see that the constraints separation can be performed using the linear algebra operations with the matrix $\mathbf{M}$ alone. Together with the Gröbner bases technique this implies full algorithmisation for computing the complete set of algebraically independent constraints and their classification.

\section{Implementation}

The above described algorithms were implemented first in Maple 1014. However, the Gröbner bases routines built-in Maple are not efficient enough to perform computation needed for the light-cone $S U(3)$ Yang-Mills mechanics (Sect.3.2). We also tried recent extensions of the Maple Gröbner bases facilities with the external packages $G b$ and $F g b$ created by J.C.Faugère [21]. Unfortunately $G b$ runs for our problems even slower than the built-in package whereas $F g b$ cannot deal with the parametric coefficients. By the last reason we cannot use yet2 the Ginv [22] software that is a $\mathrm{C}++$ module of Python and implements the efficient involutive algorithms 6 for the construction of the involutive or/and Gröbner bases.

${ }^{2}$ The implementation in Ginv of multivariate GCD computation that is necessary for computation of Gröbner bases with the parametric coefficients is in progress now in collaboration with the RWTH, Aachen. 
It should be emphasized that manipulation with the parametric coefficients is essential for the Dirac formalism due to the presence of physical parameters (e.g. masses, coupling constants) in the initial Lagrangian, the Lagrange multipliers in the total Hamiltonian (4). Having these needs in mind we implemented the algorithms in Mathematica whose built-in routine GroebnerBasis as well as Groebner in Maple allows to compute parametric Gröbner bases but performs computations much faster.

\section{Light-Cone Yang-Mills Mechanics}

Here we apply the above described scheme to a mechanical model originated from the Yang-Mills gauge field theory assuming a certain homogeneity of fields. Namely, we consider the so-called light-cone Yang-Mills mechanics which differs from the well-known instant form of Yang-Mills mechanics intensively studied during the last twenty years for a variety of reasons, both in physics and in mathematics (see e.g. 23]-33]). The alternative light-cone Yang-Mills mechanics is formulated as the light-front form version of the $S U(n)$ Yang-Mills gauge theory when the additional supposition of the gauge potentials dependence on the light-cone time only is made.

The coordinate free representation of the $S U(n)$ Yang-Mills fields action in four-dimensional Minkowski space $M_{4}$, endowed with a metric $\eta$ reads

$$
S:=\frac{1}{g_{0}^{2}} \int_{M_{4}} \operatorname{tr} F \wedge * F,
$$

where $g_{0}$ is a coupling constant and the $s u(n)$ algebra valued curvature two-form

$$
F:=d A+A \wedge A
$$

is constructed from the connection one-form $A$. The connection and curvature, as Lie algebra valued quantities are expanded in some basis $T^{a}$

$$
A=A^{a} T^{a}, \quad F=F^{a} T^{a} . \quad a=1,2, \ldots, n^{2}-1 .
$$

The metric $\eta_{\gamma \delta}$ enters the action through the dual field strength tensor $* F_{\mu \nu}:=$ $\frac{1}{2} \sqrt{-\operatorname{det} \eta} \epsilon_{\mu \nu \alpha \beta} F^{\alpha \beta}$, with the totally antisymmetric tensor $\epsilon_{\mu \nu \alpha \beta}$.

To formulate the light-cone version of the $S U(n)$ mechanics we expand the one-form $A$ in so-called light-cone basis

$$
A:=A_{+} \mathrm{d} x^{+}+A_{-} \mathrm{d} x^{-}+A_{k} \mathrm{~d} x^{k}, \quad k=1,2,
$$

where the basic one-forms $\mathrm{d} x^{ \pm}$in (13) are dual to the vectors $e_{ \pm}:=\frac{1}{\sqrt{2}}\left(e_{0} \pm e_{3}\right)$ tangent to the light-cone. The corresponding coordinates, light-cone coordinates $x^{\mu}=\left(x^{+}, x^{-}, x^{\perp}\right)$ are

$$
x^{ \pm}:=\frac{1}{\sqrt{2}}\left(x^{0} \pm x^{3}\right), \quad x^{\perp}:=x^{k}, \quad k=1,2,
$$

and non-zero components of the metric read $\eta_{+-}=\eta_{-+}=-\eta_{11}=-\eta_{22}=1$. 
Now if the components of the connection one-form $A$ in (13) are functions of the light-cone "time variable" $x^{+}$only

$$
A_{ \pm}=A_{ \pm}\left(x^{+}\right), \quad A_{k}=A_{k}\left(x^{+}\right) .
$$

the classical action (12) reduces to the following form

$$
S_{\mathrm{LC}}:=\frac{V^{(3)}}{2 g_{0}^{2}} \int \mathrm{d} x^{+}\left(F_{+-}^{a} F_{+-}^{a}+2 F_{+k}^{a} F_{-k}^{a}-F_{12}^{a} F_{12}^{a}\right) .
$$

This expression can be identified with the action of a finite dimensional model named as light-cone Yang-Mills mechanics whose dynamics is governed by the Lagrangian

$$
L:=\frac{1}{2}\left(F_{+-}^{a} F_{+-}^{a}+2 F_{+k}^{a} F_{-k}^{a}-F_{12}^{a} F_{12}^{a}\right),
$$

Deriving (15) we fix the "renormalized" coupling constant $g_{0}^{2} / V^{(3)}=1$ in (14) to simplify formulaes and use the following expression for the light-cone components of the field-strength tensor

$$
\begin{aligned}
& F_{+-}^{a}:=\frac{\partial A_{-}^{a}}{\partial x^{+}}+\mathrm{f}^{a b c} A_{+}^{b} A_{-}^{c}, \\
& F_{+k}^{a}:=\frac{\partial A_{k}^{a}}{\partial x^{+}}+\mathrm{f}^{a b c} A_{+}^{b} A_{k}^{c}, \\
& F_{-k}^{a}:=\mathrm{f}^{a b c} A_{-}^{b} A_{k}^{c}, \\
& F_{i j}^{a}:=\mathrm{f}^{a b c} A_{i}^{b} A_{j}^{c}, \quad i, j, k=1,2 .
\end{aligned}
$$

The Lagrangian (15) defines the $S U(n)$ Yang-Mills light-cone mechanics with $4\left(n^{2}-1\right)$ - degrees of freedom $A_{ \pm}, A_{k}$ evolving with respect to the light-cone time $\tau:=x^{+}$.

However due to the gauge invariance of the initial Yang-Mills theory and because in the light-cone dynamics the instant time states are given at the lightcone characteristics the corresponding evolutionary equations degenerate (see e.g. discussion in [8, 34]): not all of them are second order with respect to the light-cone time. Some of the Euler-Lagrange equations that follow from (15) represent the constraints on the variables from the extended configuration.

In the Hamiltonian description this can be seen as follows. The Legendre transformation gives the momentum $\pi_{a}^{-}$, canonically conjugated to $A_{-}^{a}$

$$
\pi_{a}^{-}:=\frac{\partial L}{\partial \dot{A_{-}^{a}}}=\dot{A_{-}^{a}}+\mathrm{f}^{a b c} A_{+}^{b} A_{-}^{c},
$$

while defining the momenta $\pi_{a}^{+}$and $\pi_{a}^{k}$ canonically conjugated to $A_{-}^{a}$ and $A_{k}^{a}$ we find the set of the primary constraints

$$
\begin{aligned}
& \varphi_{a}^{(1)}:=\pi_{a}^{+}=0 \\
& \chi_{k}^{a}:=\pi_{a}^{k}-\mathrm{f}^{a b c} A_{-}^{b} A_{k}^{c}=0 .
\end{aligned}
$$


The presence of primary constraints affects the dynamics of the degenerate system. The generic evolution is governed now by the total Hamiltonian

$$
H_{T}:=H_{C}+U_{a}(\tau) \varphi_{a}^{(1)}+V_{k}^{a}(\tau) \chi_{k}^{a},
$$

where the canonical Hamiltonian reads

$$
H_{C}=\frac{1}{2} \pi_{a}^{-} \pi_{a}^{-}-\mathrm{f}^{a b c} A_{+}^{b}\left(A_{-}^{c} \pi_{a}^{-}+A_{k}^{c} \pi_{a}^{k}\right)+\frac{1}{2} F_{12}^{a} F_{12}^{a},
$$

and $U_{a}(\tau)$ and $V_{k}^{a}(\tau)$ are the Lagrange multipliers.

Using the total Hamiltonian and the fundamental canonical Poisson brackets

$$
\left\{A_{ \pm}^{a}, \pi_{b}^{ \pm}\right\}=\delta_{b}^{a}, \quad\left\{A_{k}^{a}, \pi_{b}^{l}\right\}=\delta_{k}^{l} \delta_{b}^{a},
$$

the dynamical self-consistence of the primary constraints (16) should be checked out. From the requirement of conservation of the primary constraints $\varphi_{a}^{(1)}$ we see that

$$
0=\dot{\varphi}_{a}^{(1)}=\left\{\pi_{a}^{+}, H_{T}\right\}=\mathrm{f}^{a b c}\left(A_{-}^{b} \pi_{c}^{-}+A_{k}^{b} \pi_{c}^{k}\right),
$$

while the same procedure for the primary constraints $\chi_{k}^{a}$ gives the following self-consistency conditions

$$
0=\dot{\chi}_{k}^{a}=\left\{\chi_{k}^{a}, H_{C}\right\}-2 \mathrm{f}^{a b c} A_{-}^{b} V_{k}^{c}
$$

It is straightforward to check that the consistency conditions (18) define the $n^{2}-1$ secondary constraints $\varphi_{a}^{(2)}$

$$
\varphi_{a}^{(2)}:=\mathrm{f}_{a b c}\left(A_{-}^{b} \pi_{c}^{-}+A_{k}^{b} \pi_{c}^{k}\right)=0
$$

which obey the $s u(n)$ algebra

$$
\left\{\varphi_{a}^{(2)}, \varphi_{b}^{(2)}\right\}=\mathrm{f}_{a b c} \varphi_{c}^{(2)}
$$

However, the further analysis of the consistency conditions (19) represents not so easy tractable issue. First of all, the number of Lagrange multipliers that can be determined from (19) depends on the rank of the structure group. This can bee seen from the non-vanishing Poisson brackets between constraints $\chi_{i}^{a}$

$$
\left\{\chi_{i}^{a}, \chi_{j}^{b}\right\}=2 \mathrm{f}^{a b c} A_{-}^{c} \delta_{i j}
$$

The simplest case of the special unitary group of rank 1, the $S U(2)$ group, has been analyzed in our previous papers. The constraints analysis of the $S U(2)$ model including their separation into the first and second class can be found in 11 12 13. Below we only state these results and then discuss in more details the model with the first non-trivial rank 2 structure group, the $S U(3)$ Yang-Mills light-cone mechanics. 


\subsection{The $S U(2)$ Structure Group}

For the $s u(2)$ algebra we use the standard Pauli matrices $\sigma_{1}, \sigma_{2}, \sigma_{3}$ providing the structure constants as the totally antisymmetric three dimensional Levi-Civita symbol: $\mathrm{f}^{a b c}:=\epsilon^{a b c}, \epsilon^{123}=1$.

According to the equations (16) and (17), there are $\left(2^{2}-1\right)+\left(2^{2}-1\right) \times 2=9$ primary constraints $\varphi_{a}^{(1)}$ and $\chi_{k}^{a}$. From the consistency condition (19) for the primary constraints $\chi_{k}^{a}$ the following picture stands out

- Apart from the easy recognizable abelian constraints $\pi_{a}^{+}$and non-abelian first-class constraints $\varphi_{a}^{(2)},(20)$, there are two more constraints absent in the instant form of $S U(2)$ Yang-Mills mechanics

$$
\psi_{k}:=A_{-}^{a} \chi_{k}^{a} .
$$

Here $A_{-}^{a}$ is the null vector of the Poisson brackets $C_{a b}=\epsilon_{a b c} A_{-}^{c}$ in (21).

- The remaining four "orthogonal" constraints

$$
\chi_{k \perp}^{a}:=\chi_{k}^{a}-A_{-}^{a}\left(A_{-}^{b} \chi_{k}^{b}\right),
$$

are the second-class and satisfy the relations

$$
\begin{aligned}
& \left\{\chi_{i \perp}^{a}, \chi_{j \perp}^{b}\right\}=2 \epsilon^{a b c} A_{-}^{c} \delta_{i j}, \\
& \left\{\varphi_{a}^{(2)}, \chi_{k \perp}^{b}\right\}=\epsilon^{a b c} \chi_{k \perp}^{c} .
\end{aligned}
$$

Further analysis shows that apart from the secondary Gauss law constraints $\varphi_{a}^{2}$ there are no new constraints. Indeed, the abelian constraints $\psi_{i}$ do not create new ones

$$
\left\{\psi_{i}, H_{T}\right\}=-A_{i}^{a} \varphi_{a}^{(2)}+\pi_{a}^{-} \chi_{i}^{a}+\epsilon_{a b c} A_{i}^{a} A_{k}^{b} \chi_{k}^{c} \stackrel{\Sigma}{=} 0 .
$$

The consistency condition (19) for the "orthogonal" constraints $\chi_{i \perp}^{a}$ allows to determine the corresponding four Lagrange multiplier $V_{\perp}(\tau)$ and therefore summarizing, the $S U(2)$ light-cone Yang-Mills mechanics possesses 8 functionally independent first-class constraints $\varphi_{a}^{(1)}, \psi_{k}, \varphi_{a}^{(2)}$ and 4 second-class constraints $\chi_{k \perp}^{a}$.

\subsection{The $S U(3)$ Structure Group}

The algebraic properties of the $s u(3)$ algebra are encoded in the two independent set the skew-symmetric $\mathrm{f}_{a b c}$ and symmetric $\mathrm{d}_{a b c}$ structure constants. For the basis usually used in physical applications-the Gell-Mann basis-they are listed in the Appendix.

Since the rank of the $s u(3)$ algebra is two, the null space of the matrix $C_{a b}=$ $\mathrm{f}_{a b c} A_{-}^{c}$ is 2-dimensional. It can be spanned by two null-vectors, one linear and another one quadratic in the coordinates

$$
e_{a}^{(1)}:=A_{-}^{a}, \quad e_{a}^{(2)}:=\mathrm{d}_{a b c} A_{-}^{b} A_{-}^{c} .
$$


Using vectors $e_{a}^{(1,2)}$ we decompose the set of $2 \times\left(3^{2}-1\right)=16$ primary constraints $\chi_{k}^{a}$ as

$$
\chi_{i}^{a}=\left(\chi_{i \perp}^{a}, \psi_{i}, \varsigma_{i}\right)
$$

where

$$
\psi_{i}:=e_{a}^{(1)} \chi_{i}^{a}, \quad \varsigma_{i}:=e_{a}^{(2)} \chi_{i}^{a} .
$$

The decomposition (23) turn to be very useful owing to the special Poisson brackets relations for the decomposition components

$$
\left\{\chi_{k}^{a}, \psi_{i}\right\}=0, \quad\left\{\chi_{k}^{a}, \varsigma_{i}\right\}=0, \quad\left\{\psi_{i}, \varsigma_{k}\right\}=0, \quad\left\{\psi_{i}, \psi_{j}\right\}=0, \quad\left\{\varsigma_{i}, \varsigma_{k}\right\}=0 .
$$

The consistency conditions (19) allow to find the corresponding Lagrange multipliers $V_{k \perp}^{a}$ and to get the expressions modulo primary constraints

$$
\begin{aligned}
\left\{\psi_{i}, H_{T}\right\} & =-A_{i}^{a} \varphi_{a}^{(2)}+\text { primary constraints } \\
\left\{\varsigma_{i}, H_{T}\right\} & =\mathrm{d}_{a b c} A_{i}^{a} F_{-k}^{b} F_{-k}^{c}-2 \mathrm{~d}_{a b c} A_{-}^{a} A_{i}^{b} \varphi_{c}^{(2)}+\text { primary constraints } .
\end{aligned}
$$

According to the upper equalities (25), the constraints $\psi_{i}$ do not give rise to new secondary constraints. However, the second equation (26) states that there are two more new secondary constraints

$$
\zeta_{i}=\mathrm{d}_{a b c} A_{i}^{a} F_{-k}^{b} F_{-k}^{c} .
$$

The new constraints $\zeta_{i}$ obey the following relations:

$$
\begin{aligned}
& \left\{\zeta_{i}, \zeta_{j}\right\}=0, \\
& \left\{\psi_{i}, \zeta_{j}\right\}=\delta_{i j} \mathrm{~d}_{a b c} A_{-}^{a}\left(F_{-k}^{b} \chi_{k}^{c}-\frac{1}{2} A_{-}^{b} \varphi_{c}^{(2)}\right), \\
& \left\{\varsigma_{i}, \zeta_{j}\right\}=-\delta_{i j} \mathrm{~d}_{a b c} \mathrm{~d}_{c p q} A_{-}^{a} A_{-}^{b} F_{-k}^{p} F_{-k}^{q} .
\end{aligned}
$$

Evaluation of the right hand side in the last equations (27) by using the Gröbner basis technique (details of the basis used are given in the subsequent Sect. 4) modulo all known constraints shows that the further search for the ternary constraints terminates and from the consistency condition 3

$$
\left\{\zeta_{i}, H_{T}\right\} \stackrel{\Sigma_{2}}{=}\left\{\zeta_{i}, H_{C}\right\}+\left\{\zeta_{i}, \varsigma_{k}\right\} V_{k}^{\varsigma}
$$

one can fix two unknown functions $V_{k}^{\varsigma}$ entering the decomposition for the Lagrange multipliers $V_{k}^{a}=\left(V_{k \perp}^{a}, V_{k}^{\psi}, V_{k}^{\varsigma}\right)$.

Therefore we can now finally conclude with the statement about the complete set of constraints for the light-cone $S U(3)$ Yang-Mills mechanics. The complete set of constraints consists of 34 constraints, and among them there are

- $8+8+2=18$ first-class constraints: $\pi_{+}^{a}, \varphi_{a}^{(2)}$ and $\psi_{k}$,

- $2 \times 6+2+2=16$ second-class constraints: $\chi_{k \perp}^{a}, \varsigma_{k}$ and $\zeta_{k}$.

\footnotetext{
${ }^{3}$ The $\Sigma_{2}$ stands here for the constraint manifold defined by the primary and secondary constraints.
} 
It is worth to note here that these results are based on the tedious calculation of the Poisson bracket relations and their subsequent evaluation modulo the constraint functions using the specially constructed Gröbner basis. At the present moment, to the best of our knowledge, there is no way to overpass these straightforward calculations with a high computational complexity.

\section{Computation of the Gröbner Basis}

The goal of this section is to discuss certain properties of a Gröbner basis used in the calculation of the light-cone $S U(3)$ Yang-Mills mechanics and describe some computational aspects of its construction.

The actual calculations were performed using the the computer algebra system Mathematica (version 5.0) running on the machine 2xOpteron-242 (1.6 Ghz) with $6 \mathrm{~Gb}$ of RAM. For the simplest nontrivial case of the $S U(n)$ light-cone mechanics having the structure group $S U(2)$ we used the built-in-function GroebnerBasis with monomial order DegreeReverseLexicographic. However, for the $S U(3)$ group due to substantial increase of the number of variables as well as the number of non-vanishing structure constants $\mathrm{f}_{a b c}$ and $\mathrm{d}_{a b c}$ the memory of the above computer turns to be insufficient. To overcome this problem a special Mathematica program has been written in order to calculate the homogeneous Gröbner bases $(15$ \$10.2) allowing to use step by step the partially constructed Gröbner bases.

In doing so we built a homogeneous (grading compatible) Gröbner bases for the $S U(3)$ structure group using the grading $\Gamma$ determined by the following weights of the variables:

$$
\begin{array}{llll}
\Gamma\left(\pi_{\mu}^{a}\right)=2, & \text { for all momenta } & a=1,2, \ldots, 8, & \mu=-, 1,2, \\
\Gamma\left(A_{\mu}^{a}\right)=1, & \text { for all coordinates } & a=1,2, \ldots, 8, & \mu=-, 1,2 .
\end{array}
$$

As a monomial ordering we used a grading (degree) one with breaking ties by the following pure lexicographical order on the variables.

The order on the variables with different spatial indices was chosen as

$$
\pi_{a}^{-} \succ \pi_{b}^{1} \succ \pi_{c}^{2} \succ A_{-}^{a} \succ A_{1}^{b} \succ A_{2}^{c} \quad \text { for all } a, b, c=1,2, \ldots, 8,
$$

whereas for the variables the equal spatial indices our choice was

$$
\pi_{a}^{k} \succ \pi_{b}^{k} \succ A_{k}^{a} \succ A_{k}^{b} \quad \text { if } \quad a<b \quad \text { for any } a, b=1,2, \ldots, 8 \text {. }
$$

According to the introduced grading the $\Gamma$-degrees of the basic constraints (17), (20), (26) are given by

\begin{tabular}{c|l}
$\Gamma$-degree & Constraints \\
\hline \hline 2 & $\chi_{k}^{a}=\pi_{a}^{k}-\mathrm{f}^{a b c} A_{-}^{b} A_{k}^{c}$, \\
\hline 3 & $\varphi_{a}^{(2)}=\mathrm{f}_{a b c}\left(A_{-}^{b} \pi_{c}^{-}+A_{k}^{b} \pi_{c}^{k}\right)$, \\
\hline 5 & $\zeta_{i}=d_{a b c} A_{i}^{a} F_{-k}^{b} F_{-k}^{c} \cdot$
\end{tabular}


With such a choice of grading the constraints $\chi_{k}^{a}$ and $\varphi^{(2)}$ are the lowest degree homogeneous Gröbner basis elements $G_{2}$ and $G_{3}$ of the order 2 and 3, respectively. Higher degree elements of the basis are constructed step by step by doing the following manipulations:

(i) formation of all $S$-polynomials $\left(G_{i}, G_{j}\right)$;

(ii) elimination of some superfluous $S$-polynomials according to the Buchberger's criteria [1516 17];

(iii) computation of the normal forms of $S$-polynomials modulo the lower order elements with respect to the grading chosen.

The results of computation of the Gröbner basis elements of different orders $n$ are shown in the following table where we explicitly indicated only $S$-polynomials with non-vanishing normal form.

\begin{tabular}{l|c|l}
$G_{n}$ & Polynomials \# & Constraints and $S$-polynomials \\
\hline$G_{2}$ & 16 & $\chi_{k}^{a}$ \\
\hline$G_{3}$ & 8 & $\varphi_{a}^{(2)}$ \\
\hline$G_{4}$ & 15 & $\left(G_{3}, G_{3}\right)$ \\
\hline$G_{5}$ & 14 & $\begin{array}{l}\zeta_{i},\left(\zeta_{i}, G_{j}\right) j=2,3,4 \\
\left(G_{2}, G_{4}\right),\left(G_{3}, G_{4}\right),\left(G_{4}, G_{4}\right) \\
\end{array}$ \\
& & $\left(G_{3}, G_{3}\right)$ \\
\hline$G_{6}$ & 13 & $\left(G_{2}, G_{5}\right),\left(G_{3}, G_{5}\right),\left(G_{4}, G_{5}\right),\left(G_{5}, G_{5}\right)$ \\
& & $\left(G_{3}, G_{4}\right),\left(G_{4}, G_{4}\right)$
\end{tabular}

Our attempts to compute $G_{n}$ for $n>6$ were failed because of enormous computational expenses. It should be noted that in comparison to the $S U(3)$ Gröbner computation, the case of the $S U(2)$ structure group is computationally much more simple. In this case the construction of the complete homogeneous Gröbner basis of 64 elements takes about 60 seconds for the following lexicographic order

$$
\left\{\pi_{1}^{1}, \pi_{1}^{2}, \pi_{2}^{1}, \pi_{2}^{2}, \pi_{3}^{1}, \pi_{3}^{2}, \pi_{1}^{-}, \pi_{2}^{-}, \pi_{3}^{-}, A_{1}^{1}, A_{2}^{1}, A_{1}^{2}, A_{2}^{2}, A_{1}^{3}, A_{2}^{3}, A_{-}^{1}, A_{-}^{2}, A_{-}^{3}\right\} .
$$

\section{Concluding Remarks}

By applying the Dirac-Bergmann-Gröbner algorithm to the light-cone $S U(3)$ Yang-Mills mechanics we found the complete set of constraints and identified them in accordance with the Dirac classification as the first and the second class constraints. This was achieved by means of the exploiting the special homogeneous Gröbner basis whose components $G_{n}$ with $n \leq 6$ where computed. Though we were not able to determine the elements $G_{n}(n>6)$ of the Gröbner basis, but the knowledge of these partial components computed suffices (Sect.3.2) to deduce the complete set of constraints and categorize them. 
Performing the computation we observed that the timings in construction of polynomials of a given order may considerably vary. For instance, the element in $G_{4}$ obtained from the $S$-polynomial $\left(\varphi_{2}^{(2)}, \varphi_{3}^{(2)}\right)$ depends on the coordinates $A_{-}^{a}, A_{1}^{a}, A_{2}^{a}$ only and contains 286 terms. Its reduction requires a large number of monomial divisions at the elementary reduction steps. In contrast to that polynomial, the other polynomials in $G_{4}$ turn to be an irreducible.

Final remark, most of the total calculation time (about a month) was spend performing useless zero reductions. This is in agreement with the well-known experimental facts in practical computation of Gröbner bases for large polynomial systems 35.

\section{Acknowledgments}

We would like to thank T.Heinzl, D. McMullan, D. Mladenov and S. Krivonos for fruitful conversions on topics related to this work.

This work was supported in part by the grant No. 07-01-00660 from the Russian Foundation for Basic Research and by the grant No. 5362.2006.2. from the Ministry of Education and Science of the Russian Federation.

\section{References}

1. Pommaret, J.F.: Partial Differential Equations and Group Theory. New Perspectives for Applications. Kluwer, Dordrecht (1994)

2. Reid, G., Wittkopf, A., Boulton, A.: Reduction of systems of nonlinear partial differential equations to simplified involutive forms. Euro. J. Appl. Maths. 7, 604635 (1996)

3. Gerdt, V.P., Blinkov, Y.A.: Involutive bases of polynomial ideals. Math. Comp. Simul. 45, 519-542 (1998), arXiv:math.AC/9912027.

4. Gerdt, V.P.: Completion of linear differential systems to involution. In: Ganzha, V.G., Mayr, E.W., Vorozhtsov, E.V. (eds.) Computer Algebra in Scientific Computing / CASC'99, pp. 115-137. Springer, Berlin (1999)

5. Calmet, J., Hausdorf, M., Seiler, W.M.: A constructive introduction to involution. In: Akerkar, R. (ed.) International Symposium on Applications of Computer Algebra/ISACA'2000, pp. 33-50. Allied Publishers, New Delhi (2001)

6. Gerdt, V.P.: Involutive algorithms for computing Gröbner bases. In: Cojocaru, S., Pfister, G., Ufnarovski, V. (eds.) Computational Commutative and NonCommutative algebraic geometry. NATO Science Series, pp. 199-225. IOS Press, Amsterdam (2005), arXiv:math.AC/0501111

7. Dirac, P.A.M.: Generalized Hamiltonian dynamics, Canad. J. Math. 2, 129-148 (1950), Lectures on Quantum Mechanics, Belfer Graduate School of Science, Monographs Series, Yeshiva University, New York, 1964.

8. Sundermeyer, K.: Constrained Dynamics. Lecture Notes in Physics, vol. 169. Spinger, Heidelberg (1982)

9. Henneaux, M., Teitelboim, C.: Quantization of Gauge Systems. Princeton University Press, Princeton, New Jersey (1992)

10. Gerdt, V.P., Gogilidze, S.A.: Constrained Hamiltonian systems and Gröbner bases. In: Ganzha, V.G., Mayr, E.W., Vorozhtsov, E.V. (eds.) Computer Algebra in Scientific Computing/CASC 1999, pp. 138-146. Springer, Berlin (1999) 
11. Gerdt, V.P., Khvedelidze, A.M., Mladenov, D.M.: Analysis of constraints in lightcone version of $S U(2)$ Yang-Mills mechanics. In: Gerdt, V.P. (ed.) Computer Algebra and its Applications to Physics/CAAP'2001, Dubna, JINR, pp. 83-92 (2002), arXiv:hep-th/0209107

12. Gerdt, V.P., Khvedelidze, A.M., Mladenov, D.M.: Light-cone SU(2) Yang-Mills theory and conformal mechanics, arXiv:hep-th/0222100

13. Gerdt, V., Khvedelidze, A., Mladenov, D.: On application of involutivity analysis of differential equations to constrained dynamical systems. In: Sissakian, A.N. (ed.)Symmetries and Integrable Systems, Selected papers of the seminar, 20002005, Dubna, JINR, vol. I, pp. 132-150 (2006), arXiv:hep-th/0311174

14. Gerdt, V., Khvedelidze, A., Palii, Y.: Towards an algorithmisation of the Dirac constraint formalism. In: Calmet, J., Seiler, W.M., Tucker, R.W. (eds.) Global Integrabilty of Field Theories/GIFT' 2006, Cockroft Institute, Daresbury, UK, pp. 135-154 (2006), arXiv:math-ph/0611021

15. Becker, T., Weispfenning, V.: Gröbner Bases. A Computational Approach to Commutative Algebra. In: Graduate Texts in Mathematics, vol. 141, Springer, New York (1993)

16. Cox, D., Little, J., O'Shea, D.: Ideals, Varieties and Algorithms, 2nd edn. Springer, New York (1996)

17. Buchberger, B., Winkler, F. (eds.): Gröbner Bases and Applications. Cambridge University Press, Cambridge (1998)

18. Hurtley, D.H., Tucker, R.W., Tuckey, P.: Constrained Hamiltonian dynamics and exterior differential systems. J. Phys. A. 24, 5252-5265 (1991)

19. Seiler, W.M., Tucker, R.W.: Involution and constrained dynamics I: the Dirac approach. J. Phys. A. 28, 4431-4451 (1995)

20. Seiler, W.M.: Involution and constrained Dynamics. II: the Faddeev-Jackiw approach. J. Phys. A. 28, 7315-7331 (1995)

21. http://fgbrs.lip6.fr/salsa/Software/

22. http://invo.jinr.ru

23. Baseian, G.Z., Matinyan, S.G., Savvidy, G.K.: Nonlinear plane waves in massless Yang-Mills theory. Pisma Zh. Eksp. Teor. Fiz. 29, 641 (1979)

24. Asatryan, H.M., Savvidy, G.K.: Configuration manifold of Yang-Mills classical mechanics. Phys. Lett. A 99, 290-292 (1983)

25. Lüscher, M.: Some analytic results cocerning the mass spectrum of Yang-Mills gauge theories on a torus. Nucl. Phys. B219, 233-261 (1983)

26. Simon, B.: Some quantum operators with discrete spectrum but classically continuos spectrum. Annals of Phys. 146, 209-220 (1983)

27. Matinyan, S.G.: Dynamical chaos of nonabelian gauge fields. Fiz. Elem. Chast. Atom. Yadra 16, 522-550 (1985)

28. Soloviev, M.A.: On the geometry of classical mechanics with nonabelian gauge symmetry. Teor. Mat. Fiz. 73, 3-15 (1987)

29. Dahmen, B., Raabe, B.: Unconstrained $S U(2)$ and $S U(3)$ Yang-Mills classical mechanics. Nucl. Phys. B384, 352-380 (1992)

30. Gogilidze, S.A., Khvedelidze, A.M., Mladenov, D.M., Pavel, H.-P.: Hamiltonian reduction of $S U(2)$ Dirac-Yang-Mills mechanics. Phys. Rev. D 57, 7488-7500 (1998)

31. Khvedelidze, A.M., Pavel, H.P.: On the groundstate of Yang-Mills quantum mechanics. Phys. Lett. A 267, 96 (2000), arXiv:hep-th/9905093

32. Khvedelidze, A.M., Mladenov, D.M.: Euler-Calogero-Moser system from SU(2) Yang-Mills theory. Phys. Rev. D 62, 125016 (1-9) (2000)

33. Pavel, H.P.: SU(2) Yang-Mills quantum mechanics of spatially constant fields, arXiv:hep-th/0701283 
34. Heinzl, T.: Light-cone quantization: Foundations and applications. Lecture Notes in Physics, vol. 572, p. 55. Spinger, Heidelberg (2001)

35. Faugère, J.-C.: A new efficient algorithm for computing Gröbner bases without reduction to zero $\left(\mathrm{F}_{5}\right)$. In: Mora, T. (ed.) International Symposium on Symbolic and Algebraic Computation/ISSAC' 2002, pp. 75-83. ACM Press, New York (2002)

\section{Appendix}

The eight traceless $3 \times 3$ Hermitian Gell-Mann matrices $\lambda_{a}$ :

$$
\begin{gathered}
\lambda_{1}=\left(\begin{array}{lll}
0 & 1 & 0 \\
1 & 0 & 0 \\
0 & 0 & 0
\end{array}\right), \lambda_{2}=\left(\begin{array}{ccc}
0 & -i & 0 \\
i & 0 & 0 \\
0 & 0 & 0
\end{array}\right), \lambda_{3}=\left(\begin{array}{ccc}
1 & 0 & 0 \\
0 & -1 & 0 \\
0 & 0 & 0
\end{array}\right), \\
\lambda_{4}=\left(\begin{array}{lll}
0 & 0 & 1 \\
0 & 0 & 0 \\
1 & 0 & 0
\end{array}\right), \lambda_{5}=\left(\begin{array}{ccc}
0 & 0 & -i \\
0 & 0 & 0 \\
i & 0 & 0
\end{array}\right), \lambda_{6}=\left(\begin{array}{lll}
0 & 0 & 0 \\
0 & 0 & 1 \\
0 & 1 & 0
\end{array}\right), \\
\lambda_{7}=\left(\begin{array}{lll}
0 & 0 & 0 \\
0 & 0 & -i \\
0 & i & 0
\end{array}\right), \lambda_{8}=\frac{1}{\sqrt{3}}\left(\begin{array}{ccc}
1 & 0 & 0 \\
0 & 1 & 0 \\
0 & 0 & -2
\end{array}\right)
\end{gathered}
$$

provide a basis for the $s u(3)$ algebra

$$
\left[\lambda_{a}, \lambda_{b}\right]=2 i \sum_{c=1}^{8} \mathrm{f}_{a b c} \lambda_{c} .
$$

The nonzero structure constants $\mathrm{f}_{a b c}$, antisymmetric in all indices are listed below:

$$
\mathrm{f}_{123}=1, \quad \mathrm{f}_{147}=\mathrm{f}_{246}=\mathrm{f}_{257}=\mathrm{f}_{345}=\mathrm{f}_{516}=\mathrm{f}_{637}=1 / 2, \quad \mathrm{f}_{458}=\mathrm{f}_{678}=\sqrt{3} / 2 .
$$

The matrices $\lambda_{a}$ obey the product law

$$
\lambda_{a} \lambda_{b}=\frac{2}{3} \delta_{a b} \mathrm{I}+\sum_{c=1}^{8}\left(\mathrm{~d}_{a b c}+\imath \mathrm{f}_{a b c}\right) \lambda_{c},
$$

with the following non-vanishing values of the symmetric constants $\mathrm{d}_{a b c}$

$$
\begin{aligned}
& \mathrm{d}_{118}=\mathrm{d}_{228}=\mathrm{d}_{338}=\frac{1}{\sqrt{3}}, \quad \mathrm{~d}_{146}=\mathrm{d}_{157}=\mathrm{d}_{256}=\mathrm{d}_{344}=\mathrm{d}_{355}=\frac{1}{2}, \\
& \mathrm{~d}_{247}=\mathrm{d}_{366}=\mathrm{d}_{377}=-\frac{1}{2}, \quad \mathrm{~d}_{448}=\mathrm{d}_{558}=\mathrm{d}_{668}=\mathrm{d}_{778}=-\frac{1}{2 \sqrt{3}}, \mathrm{~d}_{888}=-\frac{1}{\sqrt{3}} .
\end{aligned}
$$

